\title{
Nonclassical-light generation in a photonic band-gap nonlinear planar waveguide
}

\author{
Jan Peřina, Jr. \\ Joint Laboratory of Optics of Palacký University and Institute of Physics of Academy of Sciences of the Czech Republic, \\ 17. listopadu 50A, 7720 07 Olomouc, Czech Republi屯 \\ Concita Sibilia, Daniela Tricca, and Mario Bertolotti \\ INFM-CNR at Dipartimento di Energetica, Università "La Sapienza" di Roma, Via A. Scarpa 16, o0161 Roma, Italy
}

\begin{abstract}
Optical parametric process occurring in a photonic band-gap planar waveguide is studied from the point of view of nonclassical-light generation. Nonlinearly interacting optical fields are described by the generalized superposition of coherent signals and noise using the method of operator linear corrections to a classical strong solution. Scattered backward-propagating fields are taken into account. Squeezed light as well as light with sub-Poissonian statistics can be obtained in two-mode fields under the specified conditions.
\end{abstract}

PACS numbers: 42.50.-p Quantum optics, 42.65.-k Nonlinear optics, 42.50.Dv Nonclassical states of the electromagnetic field, 42.65.Yj Optical parametric oscillators and amplifiers

\section{INTRODUCTION}

During the last years an increasing attention has been devoted to properties of photonic band-gap structures [1, 2]. It has been shown that intense nonlinear processes can occur in such structures built up from nonlinear materials. For example, second harmonic and sub-harmonic generation has been predicted and also observed [3, 4]. Advantages of these structures from the point of view of nonlinear interactions are based on high densities of local optical modes, on spatial localization of optical modes in confined regions of the structure and in convenient fulfilling of phase-matching conditions of a given nonlinear process. Although quantum nonlinear optics in photonic crystals is promising, investigation of these structures has been performed mostly in classical domain. Such structures are usually very short (typically tens of $\mu \mathrm{m}$ ) and so they can be conveniently used in microoptoelectronics as sources of light in near future. These nonlinear structures can also serve as sources of light with nonclassical properties, as predicted in 5]. As discussed in [6], second-harmonic-generation process in a planar nonlinear waveguide with a corrugation on the top of the waveguide can be used to control squeezing of the fundamental field; the corrugation reproduces a photonic-band gap structure. Periodicity of the grating was selected to give rise to a longitudinal confinement of the pump field, phase matching of the process was achieved introducing a spatial modulation of nonlinear susceptibility.

The aim of the paper is to investigate nonclassical properties of light (squeezing of vacuum fluctuations and sub-Poissonian photon-number statistics) generated in a nonlinear planar photonic-band-gap waveguide fabricated in such a way that a strong optical parametric pro-

*Faculty of Natural Sciences, Palacký University, 17. listopadu 50, 77207 Olomouc, Czech Republic; Electronic address: perinaj@sloup.upol.cz cess occurs inside. The corrugation of the waveguide is suitably selected in order to confine longitudinally signal and idler modes, while a weak longitudinal confinement of the pump field is assumed. Phase matching for the parametric process is achieved due to the presence of the grating and due to modal dispersion of the guided wave geometry (see, e.g., in [7]), although our considerations are not specific to a particular material.

The paper is organized as follows. Equations describing nonlinear interaction of optical fields are derived in Sec. 2. Possibility of squeezed-light generation is discussed in Sec. 3. Sec. 4 is devoted to generation of light with sub-Poissonian photon-number statistics. Sec. 5 provides conclusions. Quantum derivation of the corresponding equations is contained in Appendix A, whereas Appendix B deals with commutation relations among operators of the outgoing fields.

\section{DERIVATION OF EQUATIONS DESCRIBING THE NONLINEAR INTERACTION}

An optical field present inside a planar waveguide can be decomposed into modes. Then we have for the electric-field amplitude $\mathbf{E}(\mathbf{r}, t)$ at point $\mathbf{r}=(x, y, z)$ and time $t$ the following expression:

$$
\begin{array}{r}
\mathbf{E}(x, y, z, t)=i \sum_{m} \sqrt{\frac{\hbar \omega_{m}}{2 \epsilon_{0} \bar{\epsilon}_{r} V}} \mathbf{e}_{m} \\
\times\left[A_{m}(z) f_{m}(x, y) \exp \left[i\left(\mathbf{k}_{m}\right)_{z} z-i \omega_{m} t\right]-\text { h.c. }\right],
\end{array}
$$

where $A_{m}$ is amplitude of the $m$-th mode, $f_{m}$ means transverse eigenfunction of the $m$-th mode $\left(\Delta_{x, y} f_{m}(x, y)=0\right), \mathbf{e}_{m}$ stands for polarization vector, $\omega_{m}$ denotes frequency and $\mathbf{k}_{m}$ is wavevector of the $m$-th mode. Mean permittivity of the waveguide is denoted as $\epsilon_{r}, \epsilon_{0}$ stands for vacuum permittivity, $\hbar$ is reduced Planck constant and $V$ volume of the structure. The symbol h.c. stands for hermitian-conjugated terms. 
The electric-field amplitude $\mathbf{E}(\mathbf{r}, t)$ fulfils the wave equation inside the waveguide:

$$
\nabla^{2} \mathbf{E}-\mu \epsilon_{0} \epsilon_{r} \frac{\partial^{2} \mathbf{E}}{\partial t^{2}}=\mu \frac{\partial^{2} \mathbf{P}_{\mathrm{nl}}}{\partial t^{2}}
$$

where $\mu$ denotes vacuum permeability and $\mathbf{P}_{\mathrm{nl}}$ describes nonlinear polarization of the medium. Relative permittivity $\epsilon_{r}(\mathbf{r})$ can be written as follows:

$$
\epsilon_{r}(x, y, z)=\bar{\epsilon}_{r}(x, y)\left[1+\Delta \epsilon_{r}(x, y, z)\right] .
$$

Small variations of permeability described by $\Delta \epsilon_{r}(x, y, z)$ are related to the corrugation caused by a linear grating fabricated on the surface of the waveguide. It is useful to decompose permittivity variations $\Delta \epsilon_{r}$ as a function of spatial coordinate $z$ into harmonic functions:

$$
\Delta \epsilon_{r}(x, y, z)=\sum_{q=-\infty}^{\infty} \varepsilon_{q} \exp \left[i q \frac{2 \pi}{\Lambda_{l}} z\right], \varepsilon_{0}=0
$$

$\varepsilon_{q}$ are coefficients of the decomposition. Polarization $\mathbf{P}_{\mathrm{nl}}$ of the medium is determined using second-order susceptibility tensor $\underline{\chi}$ :

$$
\mathbf{P}_{\mathrm{nl}}=\epsilon_{0} \underline{\underline{ }} \cdot \mathbf{E} \cdot \mathbf{E}
$$

the symbol $\cdot$ stands for tensorial multiplication.

Substitution of the expression for $\mathbf{E}(\mathbf{r}, t)$ in Eq. (1) into the wave equation in Eq. (2) and the assumption $\left|\frac{\partial^{2} A_{m}}{\partial z^{2}}\right| \ll\left|\left(\mathbf{k}_{m}\right)_{z} \frac{\partial A_{m}}{\partial z}\right|$ (analog of slowly-varying envelope approximation in time domain to spatial evolution) results in the system of nonlinear differential equations for amplitudes $A_{m}$ of modes participating in the nonlinear interaction. We have six modes: signal forward-propagating mode (with amplitude $A_{s_{F}}$ ), signal backward-propagating mode $\left(A_{s_{B}}\right)$, idler forwardpropagating mode $\left(A_{i_{F}}\right)$, idler backward-propagating mode $\left(A_{i_{B}}\right)$, pump forward-propagating mode $\left(A_{p_{F}}\right)$, and finally pump backward-propagating mode $\left(A_{p_{B}}\right)$. The system of differential equations is written as follows:

$$
\begin{aligned}
\frac{d A_{s_{F}}}{d z}= & i K_{s} \exp \left(-i \delta_{s} z\right) A_{s_{B}} \\
& +2 K_{F} \exp \left(i \delta_{F} z\right) A_{p_{F}} A_{i_{F}}^{*} \\
\frac{d A_{i_{F}}}{d z}= & i K_{i} \exp \left(-i \delta_{i} z\right) A_{i_{B}} \\
& +2 K_{F} \exp \left(i \delta_{F} z\right) A_{p_{F}} A_{s_{F}}^{*} \\
\frac{d A_{s_{B}}}{d z}= & -i K_{s}^{*} \exp \left(i \delta_{s} z\right) A_{s_{F}} \\
& -2 K_{B} \exp \left(-i \delta_{B} z\right) A_{p_{B}} A_{i_{B}}^{*} \\
\frac{d A_{i_{B}}}{d z}= & -i K_{i}^{*} \exp \left(i \delta_{i} z\right) A_{i_{F}} \\
& -2 K_{B} \exp \left(-i \delta_{B} z\right) A_{p_{B}} A_{s_{B}}^{*} \\
\frac{d A_{p_{F}}=}{d z}= & -2 K_{F}^{*} \exp \left(-i \delta_{F} z\right) A_{s_{F}} A_{i_{F}} \\
\frac{d A_{p_{B}}}{d z}= & 2 K_{B}^{*} \exp \left(i \delta_{B} z\right) A_{s_{B}} A_{i_{B}}
\end{aligned}
$$

and

$$
\begin{aligned}
\delta_{a} & =\left|\left(\mathbf{k}_{a_{F}}\right)_{z}\right|+\left|\left(\mathbf{k}_{a_{B}}\right)_{z}\right|-\delta_{l}, \quad a=s, i, \\
\delta_{l} & =\frac{2 \pi}{\Lambda_{l}}, \\
\delta_{b} & =\left|\left(\mathbf{k}_{p_{b}}\right)_{z}\right|-\left|\left(\mathbf{k}_{s_{b}}\right)_{z}\right|-\left|\left(\mathbf{k}_{i_{b}}\right)_{z}\right|, \quad b=F, B .
\end{aligned}
$$

The linear coupling constants $K_{s}$ and $K_{i}$ are given as:

$$
\begin{aligned}
& K_{a}=\frac{\left|\left(\mathbf{k}_{a_{F}}\right)_{z}\right|}{2} \int d x d y \varepsilon_{1}(x, y) f_{a_{F}}^{*}(x, y) f_{a_{B}}(x, y), \\
& a=s, i .
\end{aligned}
$$

The expressions for the nonlinear coupling constants $K_{F}$ and $K_{B}$ are:

$$
\begin{aligned}
K_{b}= & \sqrt{\frac{\mu \hbar \omega_{p} \omega_{s} \omega_{i}}{8 \bar{\epsilon}_{r}^{2} V}} \int d x d y \underline{\chi} \cdot \mathbf{e}_{p} \cdot \mathbf{e}_{s} \cdot \mathbf{e}_{i} \\
& \times f_{p_{b}}(x, y) f_{s_{b}}^{*}(x, y) f_{i_{b}}^{*}(x, y), \quad b=F, B .
\end{aligned}
$$

The same equations as in Eq. (6) can be derived using quantum description of optical fields. The quantum version of Eq. (6) uses operators of electric-field amplitudes instead of classical electric-field amplitudes (for details, see Appendix A). A general solution of the quantum variant of the nonlinear system of operator equations in Eq. (6) can only be found using a completely numerical approach. However, taking into account conditions of the nonlinear interaction in a real photonic-band-gap planar waveguide, we can apply the approximation of small linear operator corrections to a classical strong solution for mean values. We can write in this case:

$$
\begin{array}{r}
\hat{A}_{a}=A_{a}+\delta \hat{A}_{a}, \\
a=s_{F}, i_{F}, p_{F}, s_{B}, i_{B}, p_{B} .
\end{array}
$$

Mean amplitudes $A_{a}$ obey classical equations given in Eq. (6), whereas the evolution of small operator corrections $\delta \hat{A}_{a}$ is governed by the following equations:

$$
\begin{aligned}
& \frac{d \delta \hat{A}_{s_{F}}}{d z}=\mathcal{K}_{s} \delta \hat{A}_{s_{B}}+\mathcal{K}_{F}\left[A_{p_{F}} \delta \hat{A}_{i_{F}}^{\dagger}+A_{i_{F}}^{*} \delta \hat{A}_{p_{F}}\right], \\
& \frac{d \delta \hat{A}_{i_{F}}}{d z}=\mathcal{K}_{i} \delta \hat{A}_{i_{B}}+\mathcal{K}_{F}\left[A_{p_{F}} \delta \hat{A}_{s_{F}}^{\dagger}+A_{s_{F}}^{*} \delta \hat{A}_{p_{F}}\right], \\
& \frac{d \delta \hat{A}_{s_{B}}}{d z}=\mathcal{K}_{s}^{*} \delta \hat{A}_{s_{F}}-\mathcal{K}_{B}\left[A_{p_{B}} \delta \hat{A}_{i_{B}}^{*}+A_{i_{B}}^{*} \delta \hat{A}_{p_{B}}\right], \\
& \frac{d \delta \hat{A}_{i_{B}}}{d z}=\mathcal{K}_{i}^{*} \delta \hat{A}_{i_{F}}-\mathcal{K}_{B}\left[A_{p_{B}} \delta \hat{A}_{s_{B}}^{\dagger}+A_{s_{B}}^{*} \delta \hat{A}_{p_{B}}\right], \\
& \frac{d \delta \hat{A}_{p_{F}}}{d z}=-\mathcal{K}_{F}^{*}\left[A_{s_{F}} \delta \hat{A}_{i_{F}}+A_{i_{F}} \delta \hat{A}_{s_{F}}\right], \\
& \frac{d \delta \hat{A}_{p_{B}}}{d z}=\mathcal{K}_{B}^{*}\left[A_{s_{B}} \delta \hat{A}_{i_{B}}+A_{i_{B}} \delta \hat{A}_{s_{B}}\right] .
\end{aligned}
$$

The constants $\mathcal{K}_{s}, \mathcal{K}_{i}, \mathcal{K}_{F}$, and $\mathcal{K}_{B}$ introduced in Eq. (11) are defined as:

$$
\begin{aligned}
\mathcal{K}_{a} & =i K_{a} \exp \left(-i \delta_{a} z\right) . \quad a=s, i, \\
\mathcal{K}_{F} & =2 K_{F} \exp \left(i \delta_{F} z\right), \\
\mathcal{K}_{B} & =2 K_{B} \exp \left(-i \delta_{B} z\right) .
\end{aligned}
$$


Assuming linear interaction being much stronger than the nonlinear one $\left(K_{F} A\right.$ and $K_{B} A$ are much lower than $K_{s}$ and $K_{i} ; A$ means an arbitrary classical amplitude), Eq. (6) can be solved analytically. We then have for the signal and idler modes:

$$
\begin{aligned}
A_{a F}(z)= & \exp \left(-i \frac{\delta_{a} z}{2}\right) \\
& \times\left[A_{a_{F}}(0) \cos \left(\Delta_{a} z\right)+\mathcal{C}_{a} \sin \left(\Delta_{a} z\right)\right], \\
A_{a B}(z)= & \exp \left(i \frac{\delta_{a} z}{2}\right) \\
\times & {\left[A_{a_{F}}(0)\left(-\frac{\delta_{a}}{2 K_{a}} \cos \left(\Delta_{a} z\right)+\frac{i \Delta_{a}}{K_{a}} \sin \left(\Delta_{a} z\right)\right)\right.} \\
& \left.+\mathcal{C}_{a}\left(-\frac{\delta_{a}}{2 K_{a}} \sin \left(\Delta_{a} z\right)-\frac{i \Delta_{a}}{K_{a}} \cos \left(\Delta_{a} z\right)\right)\right], \\
\Delta_{a}= & \sqrt{\frac{\delta_{a}^{2}}{4}-\left|K_{a}\right|^{2}}, \quad a=s, i .
\end{aligned}
$$

The symbol $A_{s_{F}}(0)\left[A_{i_{F}}(0)\right]$ stands for an incident signal[idler-] field classical amplitude. Values of the constants $\mathcal{C}_{s}$ and $\mathcal{C}_{i}$ are determined from the conditions $A_{s_{B}}(L)=0$ and $A_{i_{B}}(L)=0 ; L$ denotes the length of the waveguide.

Classical amplitudes $A_{p_{F}}$ and $A_{p_{B}}$ of the pump modes inside the waveguide are given as:

$$
\begin{aligned}
A_{p_{b}}(z) & =A_{p_{b}}(0) \\
\pm & \frac{2 K_{b}^{*} \mathcal{E}_{s} \mathcal{E}_{i}}{i\left(\Delta_{b}-\Delta_{s}-\Delta_{i}\right)}\left(\exp \left[-i\left(\Delta_{b}-\Delta_{s}-\Delta_{i}\right) z\right]-1\right) \\
\pm & \frac{2 K_{b}^{*} \mathcal{E}_{s} \mathcal{F}_{i}}{i\left(\Delta_{b}-\Delta_{s}+\Delta_{i}\right)}\left(\exp \left[-i\left(\Delta_{b}-\Delta_{s}+\Delta_{i}\right) z\right]-1\right) \\
\pm & \frac{2 K_{b}^{*} \mathcal{F}_{s} \mathcal{E}_{i}}{i\left(\Delta_{b}+\Delta_{s}-\Delta_{i}\right)}\left(\exp \left[-i\left(\Delta_{b}+\Delta_{s}-\Delta_{i}\right) z\right]-1\right) \\
\pm \frac{2 K_{b}^{*} \mathcal{F}_{s} \mathcal{F}_{i}}{i\left(\Delta_{b}+\Delta_{s}+\Delta_{i}\right)}\left(\exp \left[-i\left(\Delta_{b}+\Delta_{s}+\Delta_{i}\right) z\right]-1\right) & b=F, B .
\end{aligned}
$$

The upper signs in Eq. (14) are appropriate for $A_{p_{F}}$ whereas the lower signs stand for $A_{p_{B}}$. The constants occurring in Eq. (14) are given as:

$$
\begin{aligned}
\Delta_{F} & =\delta_{F}+\frac{\delta_{s}}{2}+\frac{\delta_{i}}{2} \\
\Delta_{B} & =-\delta_{B}-\frac{\delta_{s}}{2}-\frac{\delta_{i}}{2} \\
\mathcal{E}_{a_{F}} & =\frac{1}{2}\left(A_{a_{F}}(0)-i \mathcal{C}_{a}\right) \\
\mathcal{F}_{a_{F}} & =\frac{1}{2}\left(A_{a_{F}}(0)+i \mathcal{C}_{a}\right) \\
\mathcal{E}_{a_{B}} & =\frac{2 \Delta_{a}-\delta_{a}}{4 K_{a}}\left(A_{a_{F}}(0)-i \mathcal{C}_{a}\right) \\
\mathcal{F}_{a_{B}} & =\frac{2 \Delta_{a}+\delta_{a}}{4 K_{a}}\left(-A_{a_{F}}(0)-i \mathcal{C}_{a}\right), \quad a=s, i
\end{aligned}
$$

The constant $A_{p_{F}}(0)$ characterizes the incident pumpfield amplitude and value of the constant $A_{p_{B}}(0)$ is determined from the condition $A_{p_{B}}(L)=0$.
Solution of the system of linear equations in Eq. (11) for operator corrections can be found numerically and expressed in the matrix form:

$$
\left(\begin{array}{c}
\delta \hat{\mathcal{A}}_{F, \text { out }} \\
\delta \hat{\mathcal{A}}_{B, \text { in }}
\end{array}\right)=\left(\begin{array}{ll}
\mathcal{U}_{F F} & \mathcal{U}_{F B} \\
\mathcal{U}_{B F} & \mathcal{U}_{B B}
\end{array}\right)\left(\begin{array}{c}
\delta \hat{\mathcal{A}}_{F, \text { in }} \\
\delta \hat{\mathcal{A}}_{B, \text { out }}
\end{array}\right)
$$

where

$$
\left.\begin{array}{rl}
\delta \hat{\mathcal{A}}_{F, \text { in }}= & \left(\begin{array}{l}
\delta \hat{A}_{s_{F}}(0) \\
\delta \hat{A}_{s_{F}}^{\dagger}(0) \\
\delta \hat{A}_{i_{F}}(0) \\
\delta \hat{A}_{i_{F}}^{\dagger}(0) \\
\delta \hat{A}_{p_{F}}(0) \\
\delta \hat{A}_{p_{F}}^{\dagger}(0)
\end{array}\right), \quad \delta \hat{\mathcal{A}}_{F, \text { out }}=\left(\begin{array}{l}
\delta \hat{A}_{s_{F}}(L) \\
\delta \hat{A}_{s_{F}}^{\dagger}(L) \\
\delta \hat{A}_{i_{F}}(L) \\
\delta \hat{A}_{i_{F}}^{\dagger}(L) \\
\delta \hat{A}_{p_{F}}(L) \\
\delta \hat{A}_{p_{F}}^{\dagger}(L)
\end{array}\right), \\
\delta \hat{\mathcal{A}}_{B, \text { in }}=\left(\begin{array}{l}
\delta \hat{A}_{s_{B}}(0) \\
\delta \hat{A}_{s_{B}}^{\dagger}(0) \\
\delta \hat{A}_{i_{B}}(0) \\
\delta \hat{A}_{i_{B}}^{\dagger}(0) \\
\delta \hat{A}_{i_{B}}(L) \\
\delta \hat{A}_{i_{B}}^{\dagger}(L) \\
\delta \hat{A}_{p_{B}}(L) \\
\delta \hat{A}_{p_{B}}^{\dagger}(L)
\end{array}\right), \quad \delta \hat{\mathcal{A}}_{B, \text { out }}(1) \\
\delta \hat{A}_{p_{B}}^{\dagger}(0)
\end{array}\right)
$$

The matrices $\mathcal{U}_{F F}, \mathcal{U}_{F B}, \mathcal{U}_{B F}$, and $\mathcal{U}_{B B}$ are determined by numerical solution of Eq. (11). Precision of numerical solution of Eq. (11) can be monitored using identities stemming from commutation relations among operators (see Appendix B).

Input-output relations among linear operator corrections can be found solving Eq. (16) with respect to vectors $\delta \hat{\mathcal{A}}_{F \text {,out }}$ and $\delta \hat{\mathcal{A}}_{B \text {,out }}$ :

$$
\begin{aligned}
\left(\begin{array}{c}
\delta \hat{A}_{F, \text { out }} \\
\delta \hat{A}_{B, \text { out }}
\end{array}\right)= & \left(\begin{array}{cc}
\mathcal{U}_{F F}-\mathcal{U}_{F B} \mathcal{U}_{B B}^{-1} \mathcal{U}_{B F} & \mathcal{U}_{F B} \mathcal{U}_{B B}^{-1} \\
-\mathcal{U}_{B B}^{-1} \mathcal{U}_{B F} & \mathcal{U}_{B B}^{-1}
\end{array}\right) \\
& \times\left(\begin{array}{c}
\delta \hat{\mathcal{A}}_{F, \text { in }} \\
\delta \hat{\mathcal{A}}_{B, \text { in }}
\end{array}\right) .
\end{aligned}
$$

The output linear operator corrections contained in vectors $\delta \hat{\mathcal{A}}_{F, \text { out }}$ and $\delta \hat{\mathcal{A}}_{B \text {,out }}$ obey bosonic commutation relations provided that the input linear operator correc-

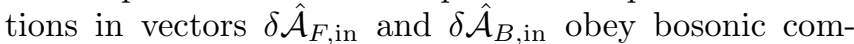
mutation relations. It has been shown in [8] that this nontrivial property is fulfilled by any system described by a quadratic hamiltonian.

Eq. (11) can be solved also iteratively assuming weak linear $\left(\mathcal{K}_{s} L \ll 1, \mathcal{K}_{i} L \ll 1\right)$ as well as nonlinear $\left(\mathcal{K}_{F} A_{p_{F}} L \ll 1, \mathcal{K}_{B} A_{p_{B}} L \ll 1\right)$ interactions among modes in the planar waveguide (weak-interaction approximation). The obtained expressions provide a useful information about the behaviour of physical quantities of interest (see the next sections).

\section{SQUEEZED-LIGHT GENERATION}

Squeezing of fluctuations of an optical field below the level characterizing vacuum fluctuations can be indicated 
by mean values of the variances of quadrature components $\hat{q}_{j}\left[\hat{q}_{j}=\hat{A}_{j}+\hat{A}_{j}^{\dagger}, \hat{A}_{j}\right.$ stands for electric-fieldamplitude operator of mode $j]$ and $\hat{p}_{j}\left[\hat{p}_{j}=-i\left(\hat{A}_{j}-\hat{A}_{j}^{\dagger}\right)\right]$ in mode $j$. Maximum amount of available squeezing measured under a suitably chosen value of the local-oscillator phase in homodyne-measurement scheme is given by principal squeeze variance $\lambda_{j}[9]$.

The above-introduced quantities can be generalized to optical fields composed of two modes; we have $\hat{q}_{j k}=$ $\hat{q}_{j}+\hat{q}_{k}$ and $\hat{p}_{j k}=\hat{p}_{j}+\hat{p}_{k}$ for quadrature components of the field composed of modes $j$ and $k$. An optical field described by a compound mode can be obtained in an output port of a beamsplitter that combines two single modes from its inputs.

We assume that the interacting fields can be described in the framework of the generalized superposition of signal and noise [10] (coherent states, squeezed states as well as noise can be considered) and then we have [1]]:

$$
\begin{aligned}
\left\langle\left(\Delta \hat{q}_{j}\right)^{2}\right\rangle= & 1+2\left[B_{j}+\operatorname{Re}\left(C_{j}\right)\right], \\
\left\langle\left(\Delta \hat{p}_{j}\right)^{2}\right\rangle= & 1+2\left[B_{j}-\operatorname{Re}\left(C_{j}\right)\right], \\
\lambda_{j}= & 1+2\left[B_{j}-\left|C_{j}\right|\right], \\
\left\langle\left(\Delta \hat{q}_{j k}\right)^{2}\right\rangle= & 2\left[1+B_{j}+B_{k}-2 \operatorname{Re}\left(\bar{D}_{j k}\right)\right. \\
& \left.+\operatorname{Re}\left(C_{j}+C_{k}+2 D_{j k}\right)\right], \\
\left\langle\left(\Delta \hat{p}_{j k}\right)^{2}\right\rangle= & 2\left[1+B_{j}+B_{k}-2 \operatorname{Re}\left(\bar{D}_{j k}\right)\right. \\
& \left.-\operatorname{Re}\left(C_{j}+C_{k}+2 D_{j k}\right)\right], \\
\lambda_{j k}= & 2\left[1+B_{j}+B_{k}-2 \operatorname{Re}\left(\bar{D}_{j k}\right)\right. \\
& \left.-\left|C_{j}+C_{k}+2 D_{j k}\right|\right] .
\end{aligned}
$$

Symbol \langle\rangle denotes quantum statistical mean value. Quantities $B_{j}, C_{j}, D_{j k}$, and $\bar{D}_{j k}$ are defined as [11]:

$$
\begin{array}{rlr}
B_{j} & =\left\langle\Delta \hat{A}_{j}^{\dagger} \Delta \hat{A}_{j}\right\rangle, & \\
C_{j} & =\left\langle\left(\Delta \hat{A}_{j}\right)^{2}\right\rangle, & \\
D_{j k} & =\left\langle\Delta \hat{A}_{j} \Delta \hat{A}_{k}\right\rangle, & j \neq k, \\
\bar{D}_{j k} & =-\left\langle\Delta \hat{A}_{j}^{\dagger} \Delta \hat{A}_{k},\right\rangle \quad j \neq k ;
\end{array}
$$

$\Delta \hat{A}_{j}=\hat{A}_{j}-\left\langle\hat{A}_{j}\right\rangle$. Quantities defined in Eq. (21) can be expressed in terms of the matrices $u$ and $v$ defined in Appendix B and using incident values of $B_{j, \text { in, } \mathcal{A}}$ and $C_{j, \text { in, } \mathcal{A}}$ related to antinormal ordering of field operators (for details, see [1]]):

$$
\begin{aligned}
B_{j, \text { in }, \mathcal{A}} & =\cosh ^{2}\left(r_{j}\right)+n_{c h, j}, \\
C_{j, \text { in }, \mathcal{A}} & =\frac{1}{2} \exp \left(i \vartheta_{j}\right) \sinh \left(2 r_{j}\right) .
\end{aligned}
$$

Symbol $r_{j}$ denotes squeeze parameter of the incident $j$-th mode, $\vartheta_{j}$ means squeeze phase, and $n_{c h, j}$ stands for mean number of incident chaotic photons. Values of $\left\langle\left(\Delta \hat{q}_{j}\right)^{2}\right\rangle$, $\left\langle\left(\Delta \hat{p}_{j}\right)^{2}\right\rangle$, and $\lambda_{j}$ less than one mean squeezing in singlemode case. Squeezed light is generated in a compoundmode (two-mode) case if values of $\left\langle\left(\Delta \hat{q}_{j k}\right)^{2}\right\rangle,\left\langle\left(\Delta \hat{p}_{j k}\right)^{2}\right\rangle$, or $\lambda_{j k}$ are less than two.

When discussing properties of the nonlinearly interacting modes we use symmetry based on the exchange of signal and idler modes. Quantities and properties that can be derived from this symmetry are not mentioned explicitly.

Discussion of squeezing is based on the investigation of principal squeeze variances because they give the maximally allowed amount of squeezing reachable in an experiment.

\section{A. Weak-interaction approximation}

Assuming incident coherent states (or vacuum states) principal squeeze variances of single modes have the following form in weak-interaction approximation:

$$
\begin{aligned}
& \lambda_{s_{F}}=1+2\left|I_{p_{F}}\right|^{2}, \\
& \lambda_{s_{B}}=1+2\left|I_{p_{B}}\right|^{2}, \\
& \lambda_{p_{F}}=1, \\
& \lambda_{p_{B}}=1,
\end{aligned}
$$

and

$$
\begin{aligned}
I_{p_{F}} & =\int_{0}^{L} d z \mathcal{K}_{F}(z) A_{p_{F}}(z), \\
I_{p_{B}} & =\int_{0}^{L} d z \mathcal{K}_{B}(z) A_{p_{B}}(z) .
\end{aligned}
$$

The expressions contained in Eq. (23) are correct up to the second power of constants $K_{s}, K_{i}, K_{F}$, and $K_{B}$. They show that principal squeeze variances of single modes are larger than one and thus no squeezing can occur in single modes.

The following expressions for the compound modes are reached under the same assumptions:

$$
\begin{aligned}
\lambda_{s_{F}, s_{B}} & =2\left[1+\left|I_{p_{F}}\right|^{2}+\left|I_{p_{B}}\right|^{2}\right] \\
\lambda_{s_{F}, i_{F}} & =2\left[1-2\left|I_{p_{F}}\right|+2\left|I_{p_{F}}\right|^{2}\right] \\
\lambda_{s_{F}, i_{B}} & =2\left[1+\left|I_{p_{F}}\right|^{2}+\left|I_{p_{B}}\right|^{2}-2\left|I_{i, p_{F}}-I_{p_{B}, s}\right|\right] \\
\lambda_{s_{B}, i_{B}} & =2\left[1-2\left|I_{p_{B}}\right|+2\left|I_{p_{B}}\right|^{2}\right] \\
\lambda_{s_{F}, p_{F}} & =2\left[1+\left|I_{p_{F}}\right|^{2}-2\left|I_{s_{F}, p_{F}}\right|\right] \\
\lambda_{s_{F}, p_{B}} & =2\left[1+\left|I_{p_{F}}\right|^{2}\right] \\
\lambda_{s_{B}, p_{B}} & =2\left[1+\left|I_{p_{B}}\right|^{2}-2\left|I_{p_{B}, s_{B}}\right|\right] \\
\lambda_{s_{B}, p_{F}} & =2\left[1+\left|I_{p_{B}}\right|^{2}\right] \\
\lambda_{p_{F}, p_{B}} & =2
\end{aligned}
$$

where

$$
\begin{aligned}
I_{i, p_{F}} & =\int_{0}^{L} d z \int_{0}^{z} d z^{\prime} \mathcal{K}_{i}^{*}(z) \mathcal{K}_{F}\left(z^{\prime}\right) A_{p_{F}}\left(z^{\prime}\right) \\
I_{p_{B}, s} & =\int_{0}^{L} d z \int_{0}^{z} d z^{\prime} \mathcal{K}_{B}(z) A_{p_{B}}(z) \mathcal{K}_{s}\left(z^{\prime}\right), \\
I_{s_{F}, p_{F}} & =\int_{0}^{L} d z \int_{0}^{z} d z^{\prime} \mathcal{K}_{F}^{*}(z) A_{s_{F}}(z) \mathcal{K}_{F}\left(z^{\prime}\right) A_{p_{F}}\left(z^{\prime}\right), \\
I_{p_{B}, s_{B}} & =\int_{0}^{L} d z \int_{0}^{z} d z^{\prime} \mathcal{K}_{B}(z) A_{p_{B}}(z) \mathcal{K}_{B}^{*}\left(z^{\prime}\right) A_{s_{B}}\left(z^{\prime}\right) .
\end{aligned}
$$


According to Eq. (25I) squeezing can occur in compound modes $\left(s_{F}, i_{F}\right)$ and $\left(s_{B}, i_{B}\right)$ owing to nonlinear process among the forward-propagating modes and that among the backward-propagating modes. Light in compound mode $\left(s_{F}, i_{B}\right)$ can be squeezed if the linear coupling (describing scattering of light in the photonic-band-gap waveguide) is stronger than the nonlinear one. As is seen from the expression for $\lambda_{s_{F}, i_{B}}$ in Eq. (25) squeezing originates in the nonlinear process and linear coupling between forward- and backward-propagating fields is inevitable for 'transfer of squeezing' into this mode. If classical amplitudes of the forward- (backward-) propagating signal mode are greater than those of the forward(backward-) propagating pump mode, squeezing can be reached in compound mode $\left(s_{F}, p_{F}\right)\left[\left(s_{B}, p_{B}\right)\right]$.

\section{B. Numerical analysis of squeezing}

Complete analysis of the behaviour of interacting modes can be reached only numerically.

We assume a strong incident forward-propagating pump field and also nonzero incident signal and idler forward-propagating fields. Squeezed light cannot be generated in single modes in this case. However, compound modes $\left(s_{F}, i_{F}\right),\left(s_{B}, i_{B}\right)$, and $\left(s_{F}, i_{B}\right)$ provide squeezed light at the output under suitably chosen values of the waveguide parameters.

Values of parameters characterizing a real nonlinear planar photonic-band-gap waveguide are assumed to lie around the point given by $L=2 \mathrm{~mm}, K_{s}=K_{i}=$ $5 \mathrm{~mm}^{-1}, K_{F}=K_{B}=5 \times 10^{-7} \mathrm{~mm}^{-1} \mathrm{mV}^{-1}$, and $A_{p_{F}}=10^{7} \mathrm{Vm}^{-1}$. That is why we concentrate our attention on quantitative analysis of behaviour of the waveguide in the vicinity of this point. We note that equality of values of linear coupling constants $K_{s}$ and $K_{i}$ can barely be found in a real waveguide but real modes can be chosen in such a way that values of these constants are nearly the same. The quantities $1 \mathrm{~mm}$ and $10^{6} \mathrm{Vm}^{-1}$ are used as appropriate units in the following graphs.

The role of length $L$ of the waveguide to squeezed-light generation is revealed in Fig. 11 We would like to remind that any change of length $L$ of the waveguide is accompanied by a little change of the period of corrugation, in order to maintain the same mode profile and the same longitudinal confinement properties of the modes. Fixing these criteria, the modes $\left(s_{F}, i_{F}\right)$ and $\left(s_{B}, i_{B}\right)$ are squeezed for any length $L$ of the structure (see Figs. [1, c). However, the maximum value of squeezing is reached for values of length $L$ around 0.5 and then there is saturation in values of squeezing. Squeezing of these modes around $20 \%$ can be reached. The optimum value of length $L$ lies around 0.3 for mode $\left(s_{F}, i_{B}\right)$ composed of one forward- and one backward-propagating field, as is shown in Fig. 1b. Squeezing in mode $\left(s_{F}, i_{B}\right)$ can occur owing to 'scattering of the already generated squeezed light' between forward- and backward-propagating fields. Saturation of the behaviour for larger values of length $L$ a)

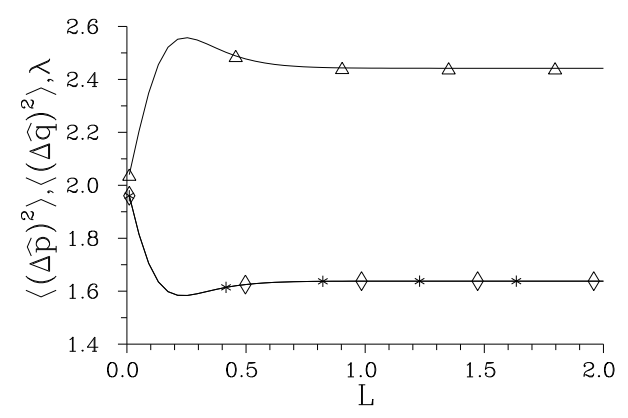

b)

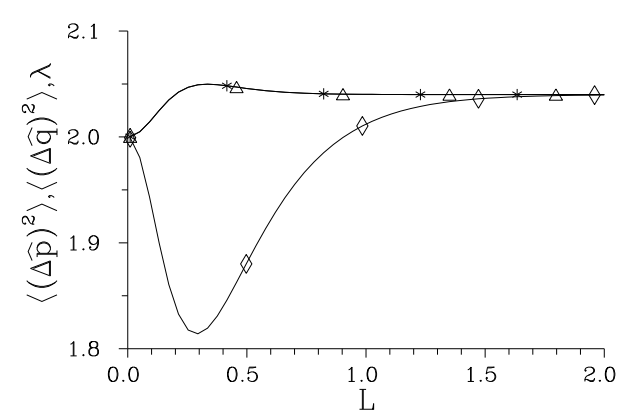

c)

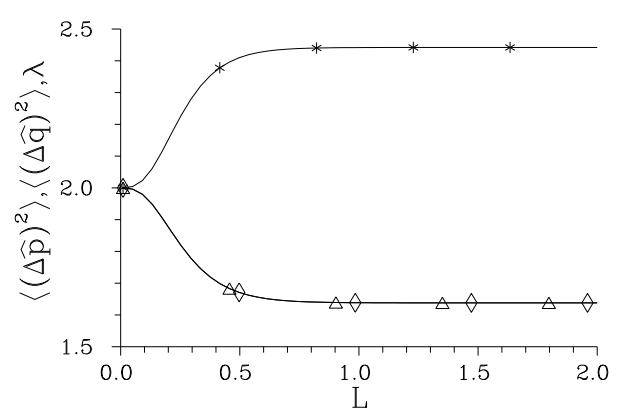

FIG. 1: Variances $\left\langle(\Delta \hat{q})^{2}\right\rangle$ (solid line with $\triangle$ ) and $\left\langle(\Delta \hat{p})^{2}\right\rangle$ $(\star)$ and principal squeeze variance $\lambda(\diamond)$ for mode $\left(s_{F}, i_{F}\right)$ (a), $\left(s_{F}, i_{B}\right)$ (b), and $\left(s_{B}, i_{B}\right)$ (c) in dependence on length $L$ of the structure; $K_{F}=K_{B}=5 \times 10^{-2}, K_{s}=K_{i}=5$, $\delta_{s}=\delta_{i}=0, \delta_{F}=\delta_{B}=0, A_{p_{F}}=10, A_{s_{F}}=A_{i_{F}}=0.1$, $A_{p_{B}}=A_{s_{B}}=A_{i_{B}}=0$, incident coherent states are assumed. Backward-propagating fields exit the waveguide at $z=0$.

is typical for interactions containing both forward- and backward-propagating fields [1].

Values of squeeze variances monotonously decrease with increasing values of nonlinear coefficients $K_{F}$ and $K_{B}$ and with increasing incident pump-pulse amplitudes $A_{p_{F}}$. This clearly shows that the origin of nonclassical properties of outgoing fields lies in the nonlinear three-mode interaction. As the graph in Fig. 22 indicates, an increase of values of nonlinear coefficient $K_{n l}$ $\left(K_{n l}=K_{F}=K_{B}\right)$ and pump-pulse amplitude $A_{p_{F}}$ two times with respect to the values characterizing the 'fixed working point' of the waveguide leads to values of squeezing around $50 \%$ for mode $\left(s_{F}, i_{F}\right)$.

Linear coupling between forward- and backward- 


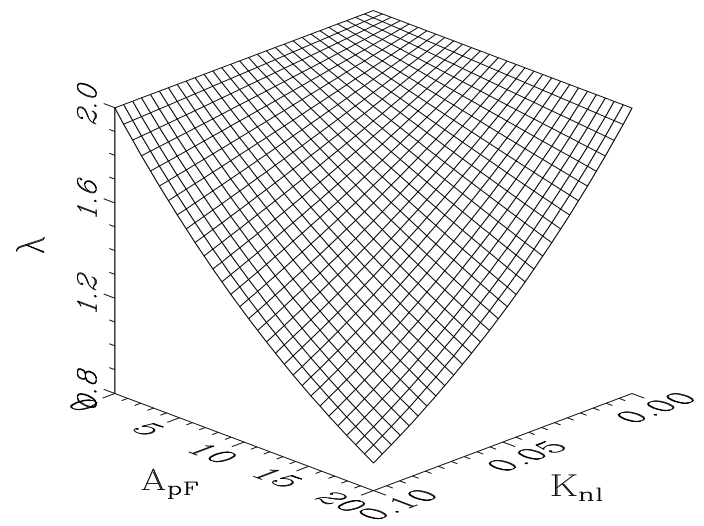

FIG. 2: Principal squeeze variance $\lambda$ of mode $\left(s_{F}, i_{F}\right)$ in dependence on nonlinear coupling constant $K_{n l}\left(K_{n l}=K_{F}=\right.$ $K_{B}$ and input pump-pulse amplitude $A_{p_{F}}(0) ; L=2$ and values of the other parameters are the same as in Fig. 1.

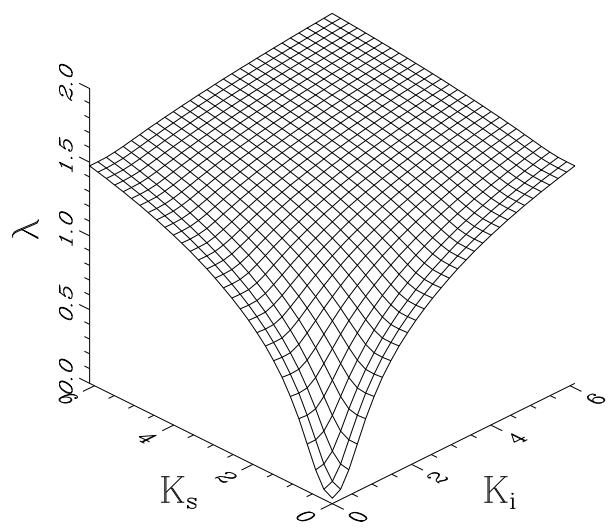

FIG. 3: Principal squeeze variance $\lambda$ of mode $\left(s_{F}, i_{F}\right)$ in dependence on linear coupling constants $K_{s}$ and $K_{i} ; L=2$ and values of the other parameters are the same as in Fig. 1.

propagating fields (described by coupling constants $K_{s}$ and $K_{i}$ ) originates in scattering of forward-propagating fields in the photonic-band-gap waveguide and does not lead to any nonclassical behaviour. Moreover, a stronger linear coupling between signal modes and idler modes suppresses squeezing, as is demonstrated in Fig. 3. It holds for mode $\left(s_{F}, i_{F}\right)$ that the larger the values of linear coupling constants the larger the values of quantities characterizing squeezing (see Fig. 31). However, nonzero values of linear coupling constants are necessary for obtaining squeezing in modes $\left(s_{F}, i_{B}\right)$ and $\left(s_{B}, i_{B}\right)$. The reason is that linear coupling enables energy transfer to backward-propagating fields (being in vacuum states at the input) from the forward-propagating fields.

Phase matching of all optical-field interactions occurring in the structure cannot be usually reached in a real structure. A typical influence of nonzero linear phase mismatch is demonstrated in Fig. 4 describing behaviour of modes $\left(s_{F}, i_{F}\right),\left(s_{F}, i_{B}\right)$, and $\left(s_{B}, i_{B}\right)$. A nonzero value of linear signal-fields phase mismatch $\delta_{s}$ causes os-

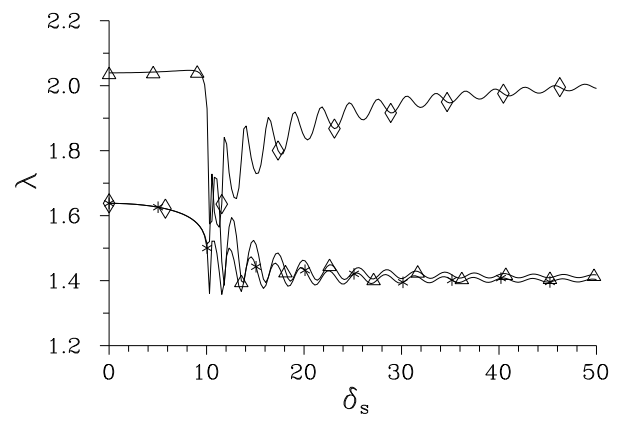

FIG. 4: Principal squeeze variance $\lambda$ for modes $\left(s_{F}, i_{F}\right)$ (solid line with $\star),\left(s_{F}, i_{B}\right)(\triangle)$, and $\left(s_{B}, i_{B}\right)(\diamond)$ in dependence on linear phase mismatch $\delta_{s} ; L=2$ and values of the other parameters are the same as in Fig. 1.

cillations in quantities characterizing squeezing. It effectively suppresses the influence of linear coupling between the signal modes and supports squeezed-light generation in mode $\left(s_{F}, i_{F}\right)$ this way. On the other hand, values of squeeze variances for mode $\left(s_{B}, i_{B}\right)$ increase with increasing values of phase mismatch $\delta_{s}$ because of lower values of amplitudes of the backward-propagating pump mode $p_{B}$. Phase relations affected by nonzero values of $\delta_{s}$ enable squeezed-light generation in mode $\left(s_{F}, i_{B}\right)$.

Increasing values of nonlinear phase mismatch $\delta_{n l}$ $\left(\delta_{n l}=\delta_{F}=\delta_{B}\right)$ result in greater values of squeeze vari-

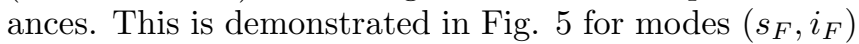
and $\left(s_{B}, i_{B}\right)$. This means that oscillations occurring along the waveguide and having their origin in nonzero values of nonlinear phase mismatch $\delta_{n l}$ effectively lower values of nonlinear coupling constants. Presence of such oscillations is visible in the behaviour of variances of quadratures $\left\langle(\Delta \hat{q})^{2}\right\rangle$ and $\left\langle(\Delta \hat{p})^{2}\right\rangle$ in mode $\left(s_{F}, i_{F}\right)$ (see Fig. $5 \mathrm{k})$. The variances belonging to mode $\left(s_{B}, i_{B}\right)$ do not show oscillations (see Fig. [5) , because oscillations are compensated in nonlinear interaction among the backward-propagating fields. Absence of such oscillations in quantities characterizing squeezing in mode $\left(s_{B}, i_{B}\right)$ indicates that squeezing in this mode has its origin only in nonlinear interaction among the backwardpropagating fields.

If we assume one of the forward-propagating signal and idler fields in vacuum state at the input (parametric downconversion) or both modes in vacuum states at the input (parametric amplification) we observe qualitatively the same behaviour of optical fields as discussed above.

If the value of an incident amplitude of either the forward-propagating signal field or forward-propagating idler field is greater than that of the pump field (parametric upconversion) squeezed light can be generated in compound modes $\left(s_{F}, i_{F}\right),\left(s_{F}, i_{B}\right)$, and $\left(s_{B}, i_{B}\right)$. Moreover, squeezed light can be reached also in compound modes $\left(s_{F}, p_{F}\right)\left[\left(i_{F}, p_{F}\right)\right]$ and $\left(s_{B}, p_{B}\right)\left[\left(i_{B}, p_{B}\right)\right]$ assum$\operatorname{ing} A_{s_{F}}>A_{p_{F}}\left[A_{i_{F}}>A_{p_{F}}\right]$. However, a strong negative influence of linear coupling affects squeezed-light gener- 
a)

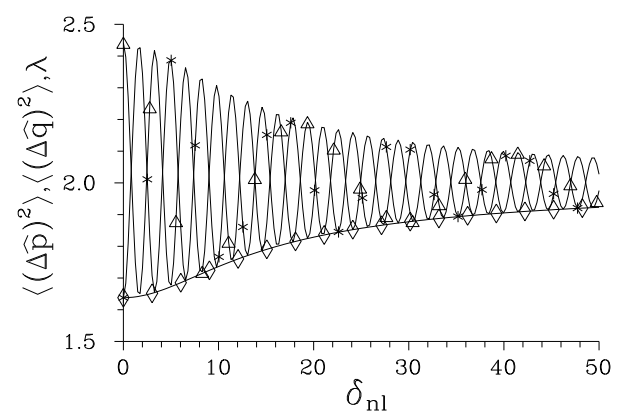

b)

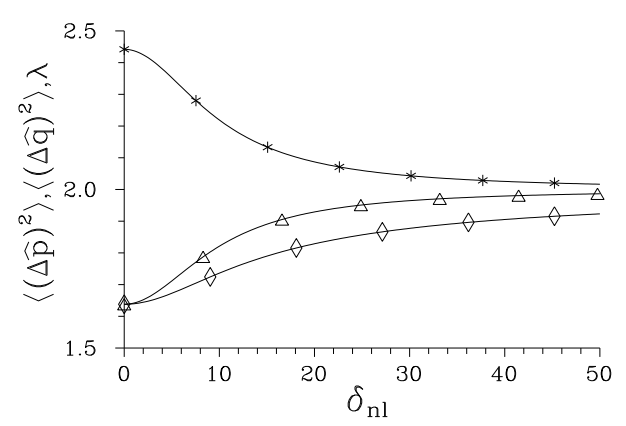

FIG. 5: Variances $\left\langle(\Delta \hat{q})^{2}\right\rangle$ (solid line with $\triangle$ ) and $\left\langle(\Delta \hat{p})^{2}\right\rangle(\star)$ and principal squeeze variance $\lambda(\diamond)$ for mode $\left(s_{F}, i_{F}\right)$ (a) and $\left(s_{B}, i_{B}\right)$ (b) in dependence on nonlinear phase mismatch $\delta_{n l}$ $\left(\delta_{n l}=\delta_{F}=\delta_{B}\right) ; L=2$ and values of the other parameters are the same as in Fig. 1.

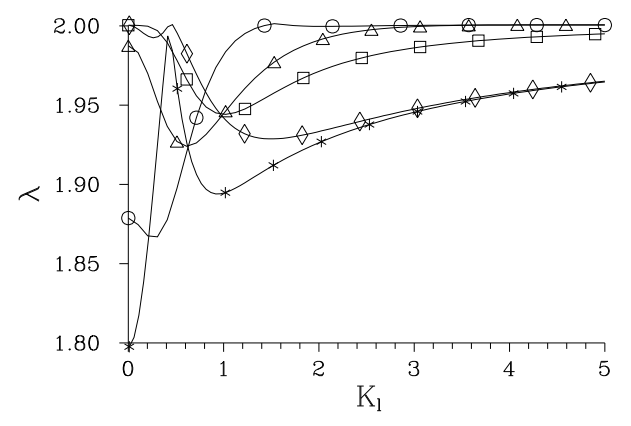

FIG. 6: Principal squeeze variance $\lambda$ of mode $\left(s_{F}, i_{F}\right)$ (solid line with $\star),\left(s_{F}, i_{B}\right)(\triangle),\left(s_{B}, i_{B}\right)(\diamond),\left(s_{F}, p_{F}\right)$ (circles), and $\left(s_{B}, p_{B}\right)$ (squares) in dependence on linear coupling constant $K_{l}\left(K_{l}=K_{s}=K_{i}\right) ; L=2, A_{s_{F}}=10, A_{i_{F}}=A_{p_{F}}=1$, values of the other parameters are the same as in Fig. 1.

ation in this regime (see Fig. 6). We note that a measurement of squeezing in a compound mode containing the pump field would require a more general scheme of homodyne detection that would use two local oscillators, one of them having the carrying frequency of the pump field, the second one oscillating at signal- or idler-field frequency.

An incident squeezed light in the forward-propagating pump mode does not support squeezed light generation. This means that values of principal squeeze variances cannot be lower than the minimum from the val-

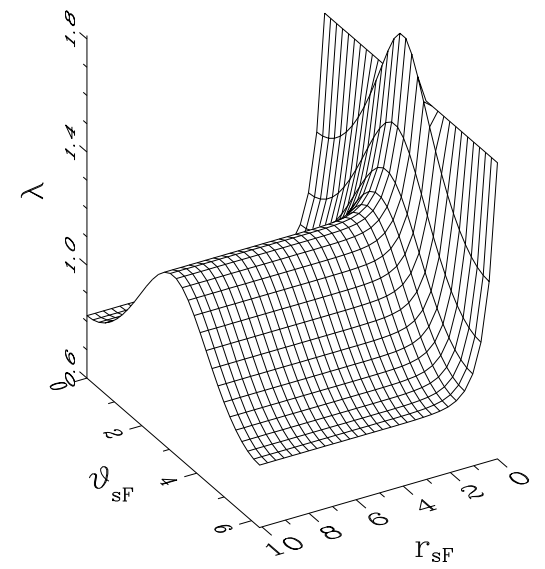

FIG. 7: Principal squeeze variance $\lambda$ of mode $\left(s_{B}, i_{B}\right)$ in dependence on squeeze parameter $r_{s F}$ and squeeze phase $\vartheta_{s F}$ characterizing the incident field; $L=2$ and values of the other parameters are the same as in Fig. 1.

ues characterizing the incident light in a given mode and those obtained assuming an unsqueezed incident forwardpropagating pump mode.

If an incident squeezed light occurs in the forwardpropagating signal mode, smaller values of squeeze variances can be reached in modes $\left(s_{B}, i_{B}\right)$ and $\left(s_{B}, i_{F}\right)$ compared to those reached with an unsqueezed input light. Values of the principle squeeze variances then depend on incident squeeze parameter $r$ and incident squeeze phase $\vartheta$ [see Fig. 7 for mode $\left.\left(s_{B}, i_{B}\right)\right]$. Values of principle squeeze variances of the other modes are greater than minimum from values characterizing the incident light and those appropriate for all unsqueezed incident beams.

\section{SUB-POISSONIAN-LIGHT GENERATION}

Statistical properties of photoelectrons emitted inside a detector reflect statistical properties of integrated intensity $\hat{W}$ of the radiation impinging on the detector. Integrated intensity $\hat{W}$ of an optical field is defined as:

$$
\hat{W}=\hat{A}^{\dagger} \hat{A},
$$

where $\hat{A}$ means an electric-field-amplitude operator of an optical field. Detection process is characterized by normally-ordered moments of integrated intensity $\hat{W}$ :

$$
\left\langle W^{k}\right\rangle_{\mathcal{N}}=\left\langle\hat{A}^{\dagger k} \hat{A}^{k}\right\rangle, \quad k=2,3, \ldots
$$

Statistical properties of photoelectrons are usually judged according to the value of Fano factor $F_{n}$ defined as:

$$
F_{n}=\frac{\left\langle(\Delta n)^{2}\right\rangle}{\langle n\rangle}=1+\frac{\left\langle(\Delta W)^{2}\right\rangle_{\mathcal{N}}}{\langle W\rangle_{\mathcal{N}}}
$$

Symbol $n$ denotes the number of photoelectrons, $\Delta n=$ $n-\langle n\rangle$, and $\Delta W=W-\langle W\rangle_{\mathcal{N}}$. We assume that intensity 
operator $\hat{W}_{i j}$ of the compound mode $(i, j)$ is determined using the relation $\hat{W}_{i j}=\hat{W}_{i}+\hat{W}_{j}$, where $\hat{W}_{i}\left(\hat{W}_{j}\right)$ denotes intensity operator of mode $i(j)$. The condition $F_{n} \geq 1$ characterizes classical fields whereas values of $F_{n}$ lower than one can be reached only for nonclassical fields (subPoissonian light). Such fields have fluctuations in the number of photoelectrons suppressed below the classical limit ( $F_{n}=1$ for a coherent state of laser radiation).

In the following we pay attention to signal and idler fields that are assumed to have intensities at singlephoton level. The planar nonlinear photonic-band-gap waveguide is pumped by a strong forward-propagating pump field. Classical amplitudes $A_{s_{F}}, A_{i_{F}}, A_{s_{B}}$, and $A_{i_{B}}$ are zero in this case. Operator amplitudes $\hat{A}$ of the signal and idler fields are then given just by their linear operator corections $\delta \hat{A}$. The quantity $10 \mathrm{Vm}^{-1}$ is used as a unit for linear amplitude corrections. Mean values of intensities using this unit are then directly equal to mean photon numbers.

\section{A. Weak-interaction approximation}

The expression for Fano factor $F_{n}$ in Eq. (29) shows that sub-Poissonian light is generated provided that $\left\langle(\Delta W)^{2}\right\rangle_{\mathcal{N}}<0$. This condition cannot be fulfilled in a single-mode case as is indicated by the following expressions valid in weak-interaction approximation and assuming coherent states with amplitudes $\xi_{j}$ for incident quantum linear corrections $\delta \hat{A}_{j}$ :

$$
\begin{aligned}
& \left\langle\left(\Delta W_{s_{F}}\right)^{2}\right\rangle_{\mathcal{N}}=2\left|I_{p_{F}}\right|^{2}\left|\xi_{s_{F}}\right|^{2}, \\
& \left\langle\left(\Delta W_{s_{B}}\right)^{2}\right\rangle_{\mathcal{N}}=2\left|I_{p_{B}}\right|^{2}\left|\xi_{s_{B}}\right|^{2} .
\end{aligned}
$$

Constants $I_{p_{F}}$ and $I_{p_{B}}$ are defined in Eq. (24). However, we arrive at the following expressions for compound modes under the same conditions:

$$
\begin{gathered}
\left\langle\left(\Delta\left(W_{s_{F}}+W_{i_{F}}\right)\right)^{2}\right\rangle_{\mathcal{N}}=4 \operatorname{Re}\left\{I_{p_{F}} \xi_{s_{F}}^{*} \xi_{i_{F}}^{*}\right\} \\
+2\left|I_{p_{F}}\right|^{2}\left(1+3\left|\xi_{s_{F}}\right|^{2}+3\left|\xi_{i_{F}}\right|^{2}\right) \\
+4 \operatorname{Re}\left\{I_{p_{F}} I_{s}^{*} \xi_{s_{B}}^{*} \xi_{i_{F}}^{*}+I_{p_{F}} I_{i} \xi_{i_{B}}^{*} \xi_{s_{F}}^{*}\right\}, \\
\left\langle\left(\Delta\left(W_{s_{F}}+W_{s_{B}}\right)\right)^{2}\right\rangle_{\mathcal{N}}= \\
2\left(\left|I_{p_{F}}\right|^{2}\left|\xi_{s_{F}}\right|^{2}+\left|I_{p_{B}}\right|^{2}\left|\xi_{s_{B}}\right|^{2}\right), \\
\left\langle\left(\Delta\left(W_{s_{F}}+W_{i_{B}}\right)\right)^{2}\right\rangle_{\mathcal{N}}= \\
2\left(\left|I_{p_{F}}\right|^{2}\left|\xi_{s_{F}}\right|^{2}+\left|I_{p_{B}}\right|^{2}\left|\xi_{i_{B}}\right|^{2}\right) \\
+4 \operatorname{Re}\left\{-I_{i, p_{F}} \xi_{s_{F}}^{*} \xi_{i_{B}}^{*}+I_{p_{B}, s} \xi_{s_{F}}^{*} \xi_{i_{B}}^{*}\right\}, \\
\left\langle\left(\Delta\left(W_{s_{B}}+W_{i_{B}}\right)\right)^{2}\right\rangle_{\mathcal{N}}=4 \operatorname{Re}\left\{I_{p_{B}} \xi_{s_{B}}^{*} \xi_{i_{B}}^{*}\right\} \\
+2\left|I_{p_{B}}\right|^{2}\left(1+3\left|\xi_{s_{B}}\right|^{2}+3\left|\xi_{i_{B}}\right|^{2}\right) \\
+4 \operatorname{Re}\left\{-I_{p_{B}} I_{s} \xi_{s_{F}}^{*} \xi_{i_{B}}^{*}-I_{p_{B}} I_{i}^{*} \xi_{i_{F}}^{*} \xi_{s_{B}}^{*}\right\} ; \\
I_{s}=\int_{0}^{L} d z K_{s}(z), \\
I_{i}=\int_{0}^{L} d z K_{i}^{*}(z) ;
\end{gathered}
$$

$I_{i, p_{F}}$ and $I_{p_{B}, s}$ are defined in Eq. (26). Analysis of expressions in Eq. (31) leads to the conclusion that negative variances $\left\langle(\Delta W)^{2}\right\rangle_{\mathcal{N}}$ of integrated intensity can occur in modes $\left(s_{F}, i_{F}\right)$ and $\left(s_{B}, i_{B}\right)$ provided that phases of the interacting fields are suitably chosen. The best conditions for sub-Poissonian-light generation in mode $\left(s_{F}, i_{F}\right)$ $\left[\left(s_{B}, i_{B}\right)\right]$ occur provided that $\arg \left(I_{p_{F}} \xi_{s_{F}}^{*} \xi_{i_{F}}^{*}\right)=\pi+2 \pi l$ $\left[\arg \left(I_{p_{B}} \xi_{s_{B}}^{*} \xi_{i_{B}}^{*}\right)=\pi+2 \pi l\right], l \in N$. The occurrence of negative variances $\left\langle(\Delta W)^{2}\right\rangle_{\mathcal{N}}$ of integrated intensity in mode $\left(s_{F}, i_{B}\right)$ requires a stronger linear interaction than the nonlinear one and again a suitably chosen phases of the interacting optical fields as can be deduced from the corresponding expression in Eq. (31).

\section{B. Numerical analysis of sub-Poissonian-light generation}

Complete analysis of sub-Poissonian behaviour of the interacting modes can be done only numerically. Provided that states of the modes can be described in the framework of generalized superposition of signal and noise [10], normally ordered moments of integrated intensity as well as photon-number distribution can be determined from parameters characterizing the state in terms of Laguerre polynomials. Details can be found in 10, 11, 12].

Numerical analysis has shown that sub-Poissonian light can be generated only in compound modes $\left(s_{F}, i_{F}\right)$, $\left(s_{F}, i_{B}\right)$, and $\left(s_{B}, i_{B}\right)$. Moreover, sub-Poissonian-light generation requires nonzero values of the incident weak forward-propagating signal and idler fields. As is demonstrated in Fig. 8 even fields containing less than one photon on average are sufficient to stimulate generation of sub-Poissonian light. Then we can have light with values of Fano factor $F_{n}$ around 0.8 in mode $\left(s_{F}, i_{F}\right)$. Scattering of signal and idler fields leads to sub-Poissonian statistics in modes $\left(s_{F}, i_{B}\right)$ and $\left(s_{B}, i_{B}\right)$. This is remarkable especially for mode $\left(s_{B}, i_{B}\right)$ being in vacuum state at the input (see Fig. 8 8 ). The larger the incident signaland idler-field amplitudes, the smaller the values of Fano factor $F_{n}$.

The possibility to generate sub-Poissonian light depends on length $L$ of the waveguide (see Fig. 9). Sub-Poissonian statistics of light in modes $\left(s_{F}, i_{F}\right)$ and $\left(s_{F}, i_{B}\right)$ occur only for shorter waveguides. On the other hand, greater values of length $L$ are convenient for sub-Poissonian statistics of light in mode $\left(s_{B}, i_{B}\right)$. Quantities characterizing this mode composed of only backward-propagating fields show saturation in dependence on length $L$ being typical for systems containing backward-propagating fields.

As is indicated by analytical expressions in Eq. (31) valid in weak-interaction approximation an efficient generation of sub-Poissonian light requires a suitable choice of phases of the forward-propagating fields. A strong influence of phase $\varphi_{s_{F}}$ of the incident forward-propagating signal field on values of Fano factor $F_{n}$ of modes $\left(s_{F}, i_{F}\right)$, 
a)

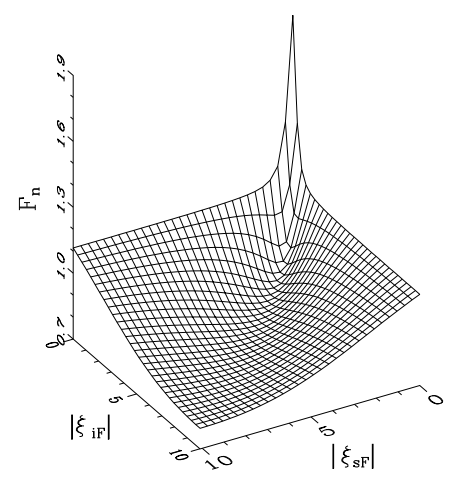

b)

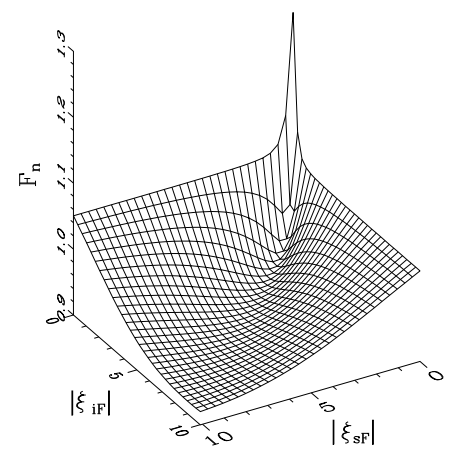

c)

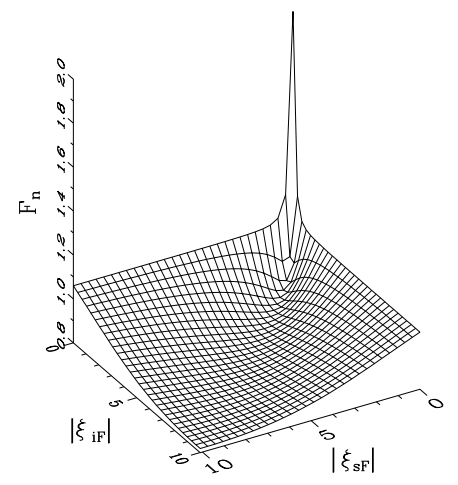

FIG. 8: Fano factor $F_{n}$ for mode $\left(s_{F}, i_{F}\right)(\mathrm{a}),\left(s_{F}, i_{B}\right)(\mathrm{b})$, and $\left(s_{B}, i_{B}\right)(\mathrm{c})$ in dependence on amplitudes $\xi_{s_{F}}$ and $\xi_{i_{F}}$; linear operator amplitude corrections $\delta \hat{A}$ are assumed to be in coherent states with amplitudes $\xi$ at the input; $K_{F}=K_{B}=$ $5 \times 10^{-2}, K_{s}=K_{i}=5, L=0.2, \delta_{s}=\delta_{i}=0, \delta_{F}=\delta_{B}=$ $0, A_{p_{F}}=10, A_{s_{F}}=A_{i_{F}}=0, A_{p_{B}}=A_{s_{B}}=A_{i_{B}}=0 ;$ $\arg \left(\xi_{s_{F}}\right)=\arg \left(\xi_{i_{F}}\right)=0, \xi_{p_{F}}=\xi_{s_{B}}=\xi_{i_{B}}=\xi_{p_{B}}=0$.

$\left(s_{F}, i_{B}\right)$, and $\left(s_{B}, i_{B}\right)$ is visualized in Fig. 10

In order to reach smaller values of Fano factor $F_{n}$ greater values of the pump field $A_{p_{F}}$ are necessary. The larger the pump amplitude $A_{p_{F}}$, the smaller the values of Fano factor $F_{n}$. For example, assuming mode $\left(s_{B}, i_{B}\right)$ $F_{n} \approx 0.9$ for $A_{p_{F}}=5$, whereas $F_{n} \approx 0.8$ for $A_{p_{F}}=10$ (see Fig. 111).

Linear coupling of modes influences sub-Poissonianlight generation as follows. The greater the values of linear coupling constants $K_{s}$ and $K_{i}$, the greater values of Fano factor $F_{n}$ are reached in mode $\left(s_{F}, i_{F}\right)$. However, even larger values of $K_{s}$ and $K_{i}$ enable to generate

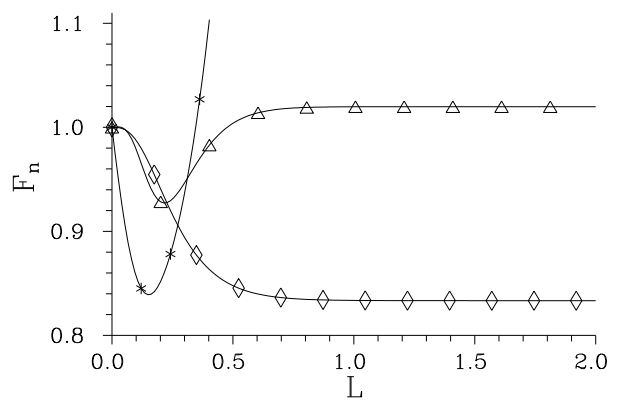

FIG. 9: Fano factor $F_{n}$ of mode $\left(s_{F}, i_{F}\right)$ (solid line with $\star$ ), $\left(s_{F}, i_{B}\right)(\triangle)$, and $\left(s_{B}, i_{B}\right)(\diamond)$ as a function of length $L ; \xi_{s_{F}}=$ $-1, \xi_{i_{F}}=1$, and values of the other parameters are the same as in Fig. 8.

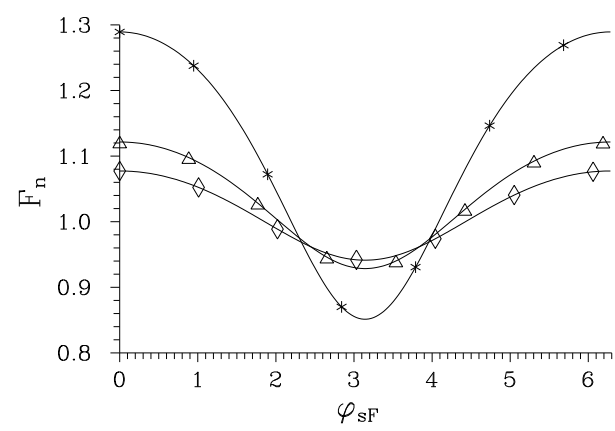

FIG. 10: Fano factor $F_{n}$ of mode $\left(s_{F}, i_{F}\right)$ (solid line with $\star$ ), $\left(s_{F}, i_{B}\right)(\triangle)$, and $\left(s_{B}, i_{B}\right)(\diamond)$ in dependence on the phase $\varphi_{s_{F}}$ $\left[\varphi_{s_{F}}=\arg \left(\xi_{s_{F}}\right)\right]$ in units of $\pi ;\left|\xi_{s_{F}}\right|=1, \xi_{i_{F}}=1$, and values of the other parameters are the same as in Fig. 8.

light with sub-Poissonian statistics. In mode $\left(s_{F}, i_{B}\right)$, greater values of $K_{s}$ result in greater values of $F_{n}$. On the other hand, greater values of $K_{i}$ lead to lower values of $F_{n}$ owing to a stronger scattering between the forwardand backward-propagating idler modes. The behaviour of mode $\left(s_{B}, i_{B}\right)$ is shown in Fig. [12 If strength of scattering between the signal and idler fields is more-less balanced $\left(K_{s} \approx K_{i}\right)$, larger values of linear coupling constants $K_{s}$ and $K_{i}$ provide smaller values of Fano factor $F_{n}$. Otherwise greater values of $K_{s}$ and $K_{i}$ neednot necessarily lead to smaller values of Fano factor $F_{n}$.

Complete phase matching of all interactions cannot be usually reached in real nonlinear photonic-band-gap waveguides. The effect of linear signal-field phase mismatch $\delta_{s}$ on values of Fano factor $F_{n}$ is relatively weak (see Fig. 131). Greater values of $\delta_{s}$ decrease values of Fano factor $F_{n}$ of mode $\left(s_{F}, i_{F}\right)$ but they increase values of Fano factor $F_{n}$ of mode $\left(s_{B}, i_{B}\right)$. This behaviour can be understood claiming that nonzero values of linear phase mismatch $\delta_{s}$ effectively weaken the linear coupling constant between the signal modes.

Nonzero values of nonlinear phase mismatches $\delta_{F}$ and $\delta_{B}$ effectively weaken nonlinear interaction in a waveguide and this acts against sub-Poissonian-light generation. As is shown in Fig. 14 sub-Poissonian light cannot be generated for greater values of $\delta_{n l}\left(\delta_{n l}=\delta_{F}=\delta_{B}\right)$ at 


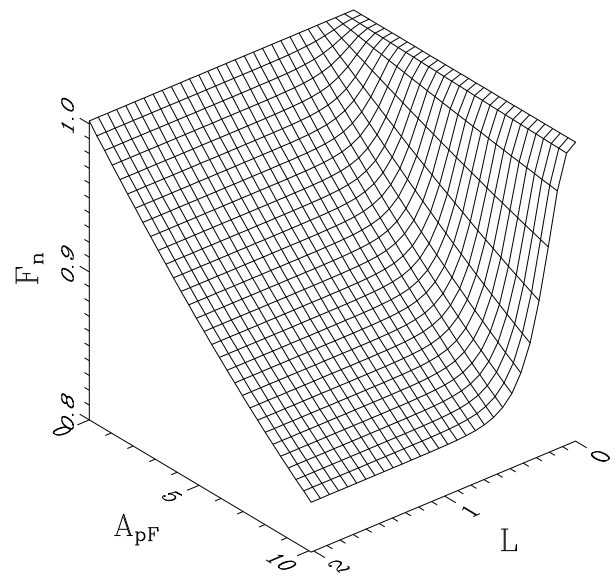

FIG. 11: Fano factor $F_{n}$ of mode $\left(s_{B}, i_{B}\right)$ in dependence on incident pump amplitude $A_{p_{F}}$ and length $L ; \xi_{s_{F}}=-1, \xi_{i_{F}}=$ 1 , and values of the other parameters are the same as in Fig. 8.

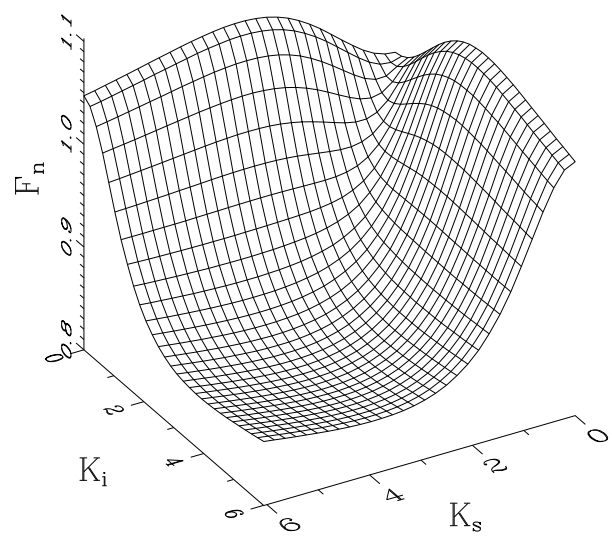

FIG. 12: Fano factor $F_{n}$ of mode $\left(s_{B}, i_{B}\right)$ in dependence on linear coupling constants $K_{s}$ and $K_{i} ; L=0.5, \xi_{s_{F}}=-1$, $\xi_{i_{F}}=1$, and values of the other parameters are the same as in Fig. 8.

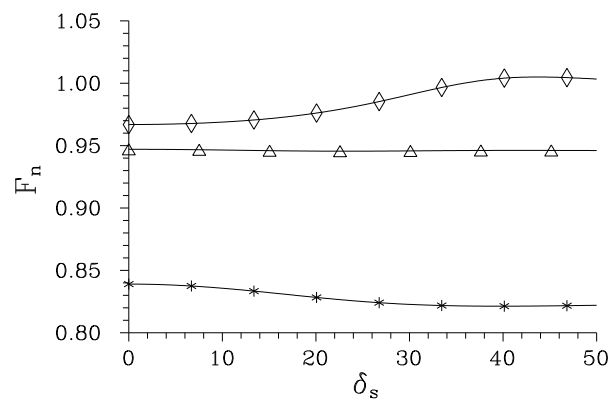

FIG. 13: Fano factor $F_{n}$ of mode $\left(s_{F}, i_{F}\right)$ (solid line with $\star$ ), $\left(s_{F}, i_{B}\right)(\triangle)$, and $\left(s_{B}, i_{B}\right)(\diamond)$ in dependence on linear phase mismatch $\delta_{s} ; \xi_{s_{F}}=-1, \xi_{i_{F}}=1$, and values of the other parameters are the same as in Fig. 8 .

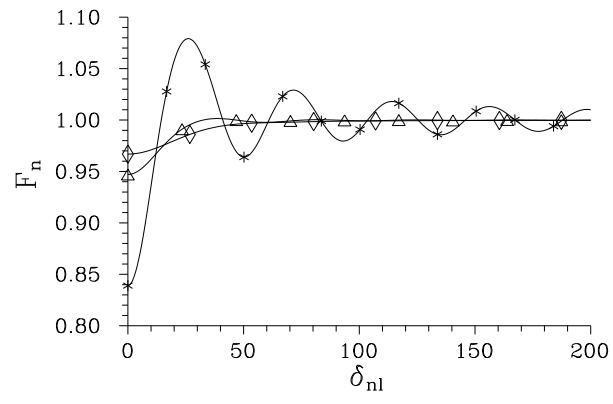

FIG. 14: Fano factor $F_{n}$ of mode $\left(s_{F}, i_{F}\right)$ (solid line with $\star),\left(s_{F}, i_{B}\right)(\triangle)$, and $\left(s_{B}, i_{B}\right)(\diamond)$ in dependence on nonlinear phase mismatch $\delta_{n l}\left(\delta_{n l}=\delta_{F}=\delta_{B}\right) ; \xi_{s_{F}}=-1, \xi_{i_{F}}=1$, and values of the other parameters are the same as in Fig. 8.

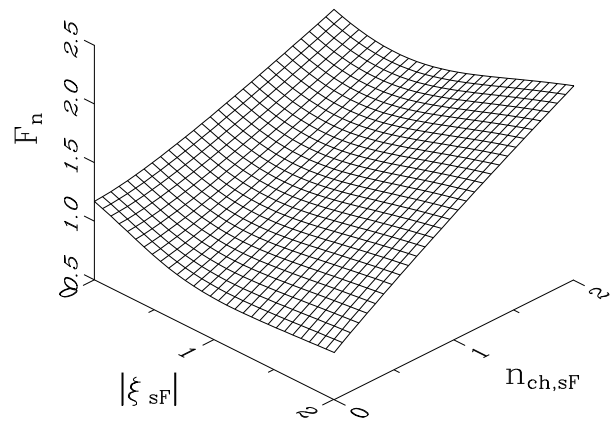

FIG. 15: Fano factor $F_{n}$ of mode $\left(s_{F}, i_{F}\right)$ in dependence on coherent amplitude $\xi_{s_{F}}$ and mean number of incident noisy photons $n_{c h, s_{F}} ; \arg \left(\xi_{s_{F}}\right)=0,, \xi_{i_{F}}=-1$, and values of the other parameters are the same as in Fig. 8.

all.

Noise present in incident fields leads to suppression of sub-Poissonian behaviour. Generation of sub-Poissonian light is rather sensitive to values of incident noise, as is shown for mode $\left(s_{F}, i_{F}\right)$ in Fig. 15]

A nonlinear planar waveguide can be also used for the suppression of incident noise. This effect has its origin in sensitivity of the nonlinear process to the phase of an incident light. To be more specific, the amplification coefficient of the incident light depends on its initial phase. If the central phase of the incident field (corresponding to a coherent signal amplitude) has the strongest amplification then the noisy part (with a blurred phase) is less amplified on average and signal-to-noise ratio increases. Reduction of the incident noise is demonstrated in Fig. [16] for single mode $s_{F}$ using second reduced moment $R_{W}$ of integrated intensity $W\left[R_{W}=\left\langle W^{2}\right\rangle_{\mathcal{N}} /\langle W\rangle_{\mathcal{N}}^{2}\right]$. Reduction of the incident noise occurs only for shorter lengths $L$ and values of incident phases in a certain region (amplification of the incident field has to occur in the waveguide). 


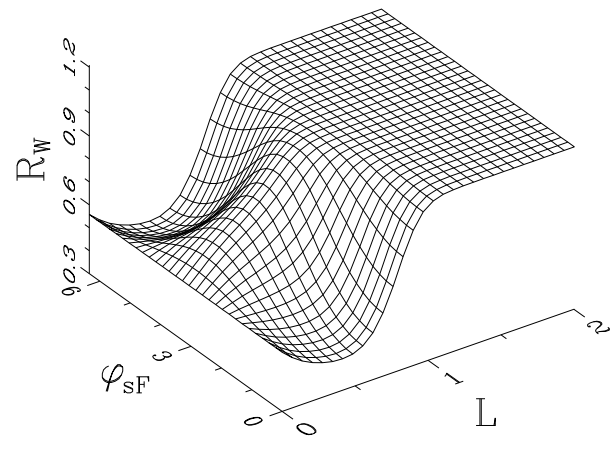

FIG. 16: Second reduced moment $R_{W}$ of integrated intensity $W$ of mode $s_{F}$ in dependence on length $L$ of the waveguide and incident phase $\varphi_{s_{F}}\left[\varphi_{s_{F}}=\arg \left(\xi_{s_{F}}\right)\right] ;\left|\xi_{s_{F}}\right|=2, \xi_{i_{F}}=2$, $n_{c h, s_{F}}=2$, and values of the other parameters are the same as in Fig. 8.

\section{CONCLUSIONS}

We have analyzed optical parametric process occurring in a nonlinear planar photonic-band-gap waveguide. It has been shown that squeezed light and light with sub-Poissonian photon-number statistics can be generated only in compound modes containing a) signal and idler forward-propagating fields, b) signal (idler) forward-propagating field and idler (signal) backwardpropagating field, and c) signal and idler backwardpropagating fields. The role of parameters characterizing a real nonlinear photonic-band-gap waveguide in generation of light with nonclassical properties has been analyzed in detail and understood. Also the influence of incident squeezed light and incident noise has been elucidated. The waveguide can be used for the suppression of incident noise. The obtained analysis will be used for the construction of a real nonlinear photonic-band-gap waveguide.

\section{Acknowledgments}

This work was supported by the COST project OC P11.003 of the Czech Ministry of Education (MŠMT) being part of the ESF project COST P11 and by grant LN00A015 of the Czech Ministry of Education. Support comming from cooperation agreement between Palacký University and University La Sapienza in Rome is acknowledged.

\section{APPENDIX A: QUANTUM DERIVATION OF THE NONLINEAR EQUATIONS}

Quantum description of nonlinearly interacting optical modes is based on the construction of momentum operator $\hat{G}(z)$ which determines Heisenberg equations of motion:

$$
\frac{d \hat{X}}{d z}=-\frac{i}{\hbar}[\hat{G}, \hat{X}]
$$

$\hat{X}$ stands for an arbitrary operator and [, ] means a commutator.

If a nonlinear interaction involves counterpropagating beams, no momentum operator can be straightforwardly assigned to Heisenberg equations as they are written in Eq.(6). However, we can proceed as follows [11]. We assume a nonlinear interaction among all involved fields as if they propagate in one direction and then we can write the momentum operator $\hat{G}(z)$ in the form:

$$
\begin{array}{r}
\hat{G}(z)=\sum_{a=s_{F}, i_{F}, p_{F}} \hbar\left(k_{a}\right)_{z} \hat{a}_{a}^{\dagger} \hat{a}_{a}+\sum_{a=s_{B}, i_{B}, p_{B}} \hbar\left(k_{a}\right)_{z} \hat{a}_{a}^{\dagger} \hat{a}_{a} \\
+\left[\hbar K_{s} \exp \left(i \delta_{l} z\right) \hat{a}_{s_{F}}^{\dagger} \hat{a}_{s_{B}}+\hbar K_{i} \exp \left(i \delta_{l} z\right) \hat{a}_{i_{F}}^{\dagger} \hat{a}_{i_{B}}+\text { h.c. }\right] \\
-\left[2 i \hbar K_{F} \hat{a}_{p_{F}} \hat{a}_{s_{F}}^{\dagger} \hat{a}_{i_{F}}^{\dagger}+2 i \hbar K_{B} \hat{a}_{p_{B}} \hat{a}_{s_{B}}^{\dagger} \hat{a}_{i_{B}}^{\dagger}+\text { h.c. }\right] .
\end{array}
$$

We now substitute the creation operators $\left(\hat{a}^{\dagger}\right)$ of the backward-propagating fields by newly introduced fictions annihilation operators $(\hat{b})$ and vice versa, i.e.

$$
\begin{aligned}
& \hat{a}_{s_{B}}^{\dagger} \leftarrow \hat{b}_{s_{B}}, \hat{a}_{i_{B}}^{\dagger} \leftarrow \hat{b}_{i_{B}}, \hat{a}_{p_{B}}^{\dagger} \leftarrow \hat{b}_{p_{B}}, \\
& \hat{a}_{s_{B}} \leftarrow \hat{b}_{s_{B}}^{\dagger}, \hat{a}_{i_{B}} \leftarrow \hat{b}_{i_{B}}^{\dagger}, \hat{a}_{p_{B}} \leftarrow \hat{b}_{p_{B}}^{\dagger} .
\end{aligned}
$$

Heisenberg equations in Eq. (A1) then have the form:

$$
\begin{aligned}
\frac{d \hat{a}_{s_{F}}}{d z}= & i\left(k_{s_{F}}\right)_{z} \hat{a}_{s_{F}}+i K_{s} \exp \left(i \delta_{l} z\right) \hat{b}_{s_{B}}^{\dagger} \\
& +2 K_{F} \hat{a}_{p_{F}} \hat{a}_{i_{F}}^{\dagger}, \\
\frac{d \hat{a}_{i_{F}}}{d z}= & i\left(k_{i_{F}}\right)_{z} \hat{a}_{i_{F}}+i K_{i} \exp \left(i \delta_{l} z\right) \hat{b}_{i_{B}}^{\dagger} \\
& +2 K_{F} \hat{a}_{p_{F}} \hat{a}_{s_{F}}^{\dagger}, \\
\frac{d \hat{b}_{s_{B}}^{\dagger}=}{d z}= & -i\left(k_{s_{B}}\right)_{z} \hat{b}_{s_{B}}^{\dagger}-i K_{s}^{*} \exp \left(-i \delta_{l} z\right) \hat{a}_{s_{F}} \\
& -2 K_{B} \hat{b}_{p_{B}}^{\dagger} \hat{b}_{i_{B}}, \\
\frac{d \hat{b}_{i_{B}}^{\dagger}=}{d z}-i\left(k_{i_{B}}\right)_{z} \hat{b}_{i_{B}}^{\dagger}-i K_{i}^{*} \exp \left(-i \delta_{l} z\right) \hat{a}_{i_{F}} & -2 K_{B} \hat{b}_{p_{B}}^{\dagger} \hat{b}_{s_{B}}, \\
\frac{d \hat{a}_{p_{F}}=}{d z}= & i\left(k_{p_{F}}\right)_{z} \hat{a}_{p_{F}}-2 K_{F}^{*} \hat{a}_{s_{F}} \hat{a}_{i_{F}}, \\
\frac{d \hat{b}_{p_{B}}^{\dagger}=}{d z}= & -i\left(k_{p_{B}}\right)_{z} \hat{b}_{p_{B}}^{\dagger}+2 K_{B}^{*} \hat{b}_{s_{B}}^{\dagger} \hat{b}_{i_{B}}^{\dagger} .
\end{aligned}
$$

Returning to the original operators $\hat{a}^{\dagger}, \hat{a}$ in Eq. (A4) using substitution in Eq. (A3) and transforming Eq. (A4) into interaction picture $\left(\hat{a}_{a}(z)=\hat{A}_{a}(z) \exp \left[i\left(k_{a}\right)_{z} z\right]\right)$ we arrive at the system of equations written in Eq. (6). We only have electric-field-amplitude operators instead of classical electric-field amplitudes occurring in Eq. (6). 
Quantum interpretation of the nonlinear process of three mode interaction at single-photon level then immediately provides the following conservation law of the overall number of photons in the interaction:

$$
\begin{gathered}
\frac{d}{d z}\left\langle\hat{A}_{s_{F}}^{\dagger} \hat{A}_{s_{F}}+\hat{A}_{i_{F}}^{\dagger} \hat{A}_{i_{F}}+2 \hat{A}_{p_{F}}^{\dagger} \hat{A}_{p_{F}}\right. \\
\left.-\hat{A}_{s_{B}}^{\dagger} \hat{A}_{s_{B}}-\hat{A}_{i_{B}}^{\dagger} \hat{A}_{i_{B}}-2 \hat{A}_{p_{B}}^{\dagger} \hat{A}_{p_{B}}\right\rangle=0 .
\end{gathered}
$$

The symbol $\langle>$ denotes mean quantum statistical value.

\section{APPENDIX B: IDENTITIES OBEYED BY A SOLUTION OF EQ. (11)}

Solution of Eq. (11) for the annihilation operators of linear operator corrections can be expressed as:

$$
\begin{aligned}
\left(\begin{array}{c}
\delta \hat{A}(L) \\
\delta \hat{B}^{\dagger}(L)
\end{array}\right)= & \left(\begin{array}{ll}
u_{11} & u_{12} \\
u_{21} & u_{22}
\end{array}\right)\left(\begin{array}{c}
\delta \hat{A}(0) \\
\delta \hat{B}^{\dagger}(0)
\end{array}\right) \\
& +\left(\begin{array}{ll}
v_{11} & v_{12} \\
v_{21} & v_{22}
\end{array}\right)\left(\begin{array}{c}
\delta \hat{A}^{\dagger}(0) \\
\delta \hat{B}(0)
\end{array}\right)
\end{aligned}
$$

and

$$
\delta \hat{A}(z)=\left(\begin{array}{l}
\delta \hat{A}_{s_{F}}(z) \\
\delta \hat{A}_{i_{F}}(z) \\
\delta \hat{A}_{p_{F}}(z)
\end{array}\right), \quad \delta \hat{B}^{\dagger}(z)=\left(\begin{array}{l}
\delta \hat{A}_{s_{B}}(z) \\
\delta \hat{A}_{i_{B}}(z) \\
\delta \hat{A}_{p_{B}}(z)
\end{array}\right)
$$

Quantum method of the derivation of Eq. (6) described in Appendix A indicates that the following commutation relations have to be fulfilled:

$$
\begin{aligned}
& {\left[\delta \hat{A}_{i}(L), \delta \hat{A}_{k}(L)\right]=0,} \\
& {\left[\delta \hat{A}_{i}(L), \delta \hat{A}_{k}^{\dagger}(L)\right]=\delta_{i k},} \\
& {\left[\delta \hat{A}_{i}(L), \delta \hat{B}_{k}(L)\right]=0,}
\end{aligned}
$$

$$
\begin{aligned}
{\left[\delta \hat{A}_{i}(L), \delta \hat{B}_{k}^{\dagger}(L)\right] } & =0 \\
{\left[\delta \hat{B}_{i}^{\dagger}(L), \delta \hat{B}_{k}(L)\right] } & =-\delta_{i k}, \\
{\left[\delta \hat{B}_{i}^{\dagger}(L), \delta \hat{B}_{k}^{\dagger}(L)\right] } & =0 .
\end{aligned}
$$

Substitution of expressions in Eq. (B1) into relations in Eq. (B3) provides identities for the matrices $u$ and $v$ defined in Eq. (B1):

$$
\begin{array}{r}
\sum_{j}\left[\left(u_{11}\right)_{i j}\left(v_{11}\right)_{k j}-\left(u_{12}\right)_{i j}\left(v_{12}\right)_{k j}-\left(v_{11}\right)_{i j}\left(u_{11}\right)_{k j}\right. \\
\left.+\left(v_{12}\right)_{i j}\left(u_{12}\right)_{k j}\right]=0 \\
\sum_{j}\left[\left(u_{11}\right)_{i j}\left(u_{11}^{*}\right)_{k j}-\left(u_{12}\right)_{i j}\left(u_{12}^{*}\right)_{k j}-\left(v_{11}\right)_{i j}\left(v_{11}^{*}\right)_{k j}\right. \\
\left.+\left(v_{12}\right)_{i j}\left(v_{12}^{*}\right)_{k j}\right]=\delta_{i k}, \\
\sum_{j}\left[\left(u_{11}\right)_{i j}\left(u_{21}^{*}\right)_{k j}-\left(u_{12}\right)_{i j}\left(u_{22}^{*}\right)_{k j}-\left(v_{11}\right)_{i j}\left(v_{21}^{*}\right)_{k j}\right. \\
\left.+\left(v_{12}\right)_{i j}\left(v_{22}^{*}\right)_{k j}\right]=0 \\
\sum_{j}\left[\left(u_{11}\right)_{i j}\left(v_{21}\right)_{k j}-\left(u_{12}\right)_{i j}\left(v_{22}\right)_{k j}-\left(v_{11}\right)_{i j}\left(u_{21}\right)_{k j}\right. \\
\left.+\left(v_{12}\right)_{i j}\left(u_{22}\right)_{k j}\right]=0 \\
\sum_{j}\left[\left(u_{21}\right)_{i j}\left(u_{21}^{*}\right)_{k j}-\left(u_{22}\right)_{i j}\left(u_{22}^{*}\right)_{k j}-\left(v_{21}\right)_{i j}\left(v_{21}^{*}\right)_{k j}\right. \\
\left.+\left(v_{22}\right)_{i j}\left(v_{22}^{*}\right)_{k j}\right]=-\delta_{i k}, \\
\sum_{j}\left[\left(u_{21}\right)_{i j}\left(v_{21}\right)_{k j}-\left(u_{22}\right)_{i j}\left(v_{22}\right)_{k j}-\left(v_{21}\right)_{i j}\left(u_{21}\right)_{k j}\right. \\
\left.+\left(v_{22}\right)_{i j}\left(u_{22}\right)_{k j}\right]=0 .
\end{array}
$$

Identities given in Eq. (B4) are useful for controlling precision of numerical integration of the corresponding differential equations.
[1] M.Bertolotti, C.M. Bowden, and C.Sibilia, Nanoscale Linear and Nonlinear Optics, AIP Vol. 560 (AIP,2001).

[2] J.D. Joannopoulos, R.D. Meade, and J.N. Winn, Photonic Crystals: Molding the Flow of Light (Princeton University Press, Princeton, 1995).

[3] M. Scalora, M.J. Bloemer, A.S. Manka, J. P. Bowling, C.M. Bowden, R. Viswanathan, and J. W. Haus, Phys. Rev. A 56, 3166 (1997).

[4] Y. Dumeige, P. Vidakovic, S. Sauvage, I. Sagnes, J.A. Levenson, C. Sibilia, M. Centini, G. D'Aguanno, and M. Scalora, Appl. Phys. Lett. 78, 3021 (2001).

[5] K. Sakoda, J. Opt. Soc. Am. B 19, 2060 (2002).

[6] D.Tricca, C.Sibilia, S.Severini, M.Bertolotti, M.Scalora, C.M. Bowden, and K.Sakoda, J. Opt. Soc. Am. B 21,
671 (2004).

[7] I. Cristiani, G.P. Banfi, V. Degiorgio, and L. Tartara, Applied Phys. Lett. 75, 1198 (1999).

[8] A. Luis and J. Peřina, Quantum Semiclass. Opt. 8, 39 (1996).

[9] A. Lukš, V. Peřinová, and J. Peřina, Opt. Commun. 67, 149 (1988).

[10] J. Peřina, Quantum Statistics of Linear and Nonlinear Optical Phenomena (Kluwer, Dordrecht, 1991).

[11] J. Peřina Jr. and J. Peřina, Progress in Optics 41, Ed. E. Wolf, (Elsevier Science, Amsterdam, 2000), p. 362.

[12] V. Peřinová, Optica Acta 28, 747 (1981); V. Peřinová and J. Peřina, Optica Acta 28, 769 (1981). 